\title{
Spin-Orbit Angles: Reflections of Dynamics in Systems
}

\author{
A.H.M.J. Triaud ${ }^{1}$, D. Queloz ${ }^{1} \&$ A.C. Cameron ${ }^{2}$ \\ ${ }^{1}$ Observatoire Astronomique de l'Université de Genève, Chemin des Maillettes \\ 51,CH-1290 Sauverny, Switzerland [Amaury.Triaud@unige.ch] \\ ${ }^{2}$ SUPA, School of Physics \& Astronomy, University of St Andrews, North Haugh, \\ KY16 9SS, St Andrews, Fife, Scotland, UK
}

\begin{abstract}
We will present our campaign to estimate the projected spin-orbit angle for transiting hot Jupiters, measured by observing the Rossiter-McLaughlin effect. Combining our efforts to those of other teams we show what the current distribution in projected spin-orbit angle is. Following this we elaborate on how this compares to migratory theories in conjunction with other pieces of evidence.
\end{abstract}

\section{Context}

Prior to the discovery of the first extrasolar planet found by Mayor \& Queloz (1995), it was in general expected that giant planets would not be found closer to their parent star than Jupiter is. This discovery called for a migrating mechanism to bring a gas giant from its birth location to the currently observed one. Lin, Bodenheimer \& Richardson (1996) using work from Golreich \& Tremaine (1979) presented the idea that planets ought to migrate by exchanging angular momentum with the protoplanetary disc. An alternative explanation came with Rasio \& Ford (1996) whereby in an unstable multiplanetary system, planets would gradually scatter each others, some arriving on close orbits.

The discovery of $51 \mathrm{Peg} \mathrm{b}$ and the other hot Jupiters shifted the generally accepted view of well ordered, hierarchical systems such as our own towards more diverse systems. Indeed, with masses ranging over several orders of magnitude, very different radii, large eccentricities and a curious period distribution, planets have demonstrated an enormous variety in their observed parameters.

More recently a new observable appeared: the projected spin-orbit angle $\beta$ (also called $\lambda$ ) which is obtained via the Rossiter-McLaughlin effect. First theorised by Holt (1893) as a way to determine stellar rotation, it was observed by Rossiter (1924) and McLaughlin (1924) on eclipsing binaries. In the standard case where the secondary would orbit with an orbital spin parallel to the primary's rotation axis, one would observe an anomalous red-shift in radial velocities as the secondary first eclipses the advancing, blue-shifted, hemisphere of the primary. As the secondary moves over the primary's redhifted, receding, hemisphere, radial velocities would show an anomalous blue-shift. It was first observed by Queloz et al. (2000) for HD 209458b and on an increasing number of transiting hot Jupiters since, most systems appearing aligned 
with their star (see Josh Winn and Guillaume Hébrard's contributions and citations within).

\section{The HARPS Rossiter-McLaughlin survey}
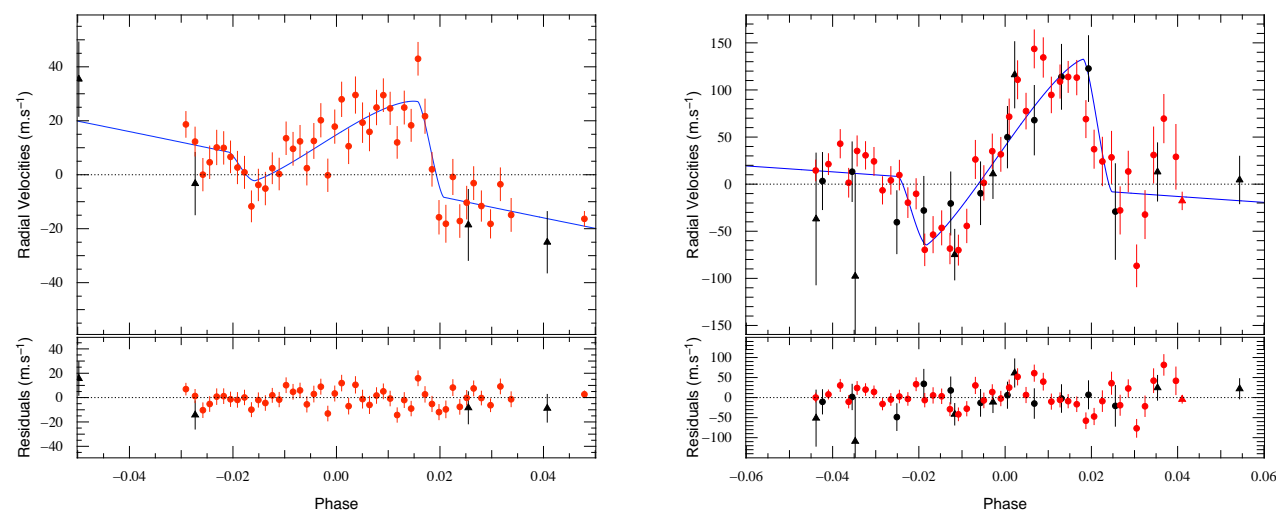

Figure 1: The Rossiter-McLaughlin effect for WASP-15b and WASP-17b and residuals as appearing in Triaud et al. (2010). Red symbols are HARPS observations, black symbols are CORALIE observations.

The WASP consortium has two instruments located in La Palma, Canary Islands, Spain, and in Sutherland, South Africa. The aim is to discover transiting planets (see contributions by Coel Hellier and Francesca Faedi). WASP produces candidates which are then confirmed on the $193 \mathrm{~cm}$ at OHP, the NOT at La Palma, and for the southern candidates, by the CORALIE spectrograph on the $1.2 \mathrm{~m}$ Swiss Euler Telescope at La Silla, Chile. After confirming some of those candidates as being planets we use the HARPS spectrograph, mounted on the ESO $3.6 \mathrm{~m}$, also at La Silla, to refine the orbits but also to get high signal to noise and high cadence observations during the transits of the WASP planets.

Our first batch of 8 observations was published in three papers: Gillon et al. (2009), Queloz et al. (2010) and Triaud et al. (2010). On these eight we found that four of our targets were retrograde: WASP-2b, 8b, 15b and 17b (see Fig. 1), an unusually large number compared to other results in the literature. We therefore attempted a statistical analysis of all the Rossiter-McLaughlin effect known then: 26, trying to see which of the published theoretical predictions could be compared to the observations (see Triaud et al. (2010)).

Figure 2 shows the cumulative distribution of projected spin orbit angles, compared with predictions by Fabrycky \& Tremaine (2007) and by Nagasawa, Ida \& Bessho (2008) which were the two predictions displaying the largest range of angles. We see that predictions by Fabrycky \& Tremaine (2007) fit the best. These authors used the Kozai mechanism caused by an outer binary with tidal friction to produce their results while Nagasawa et al. (2008) used planet-planet scattering, initiating Kozai cycles, and tidal friction. Both used dynamical events followed by tidal friction. 


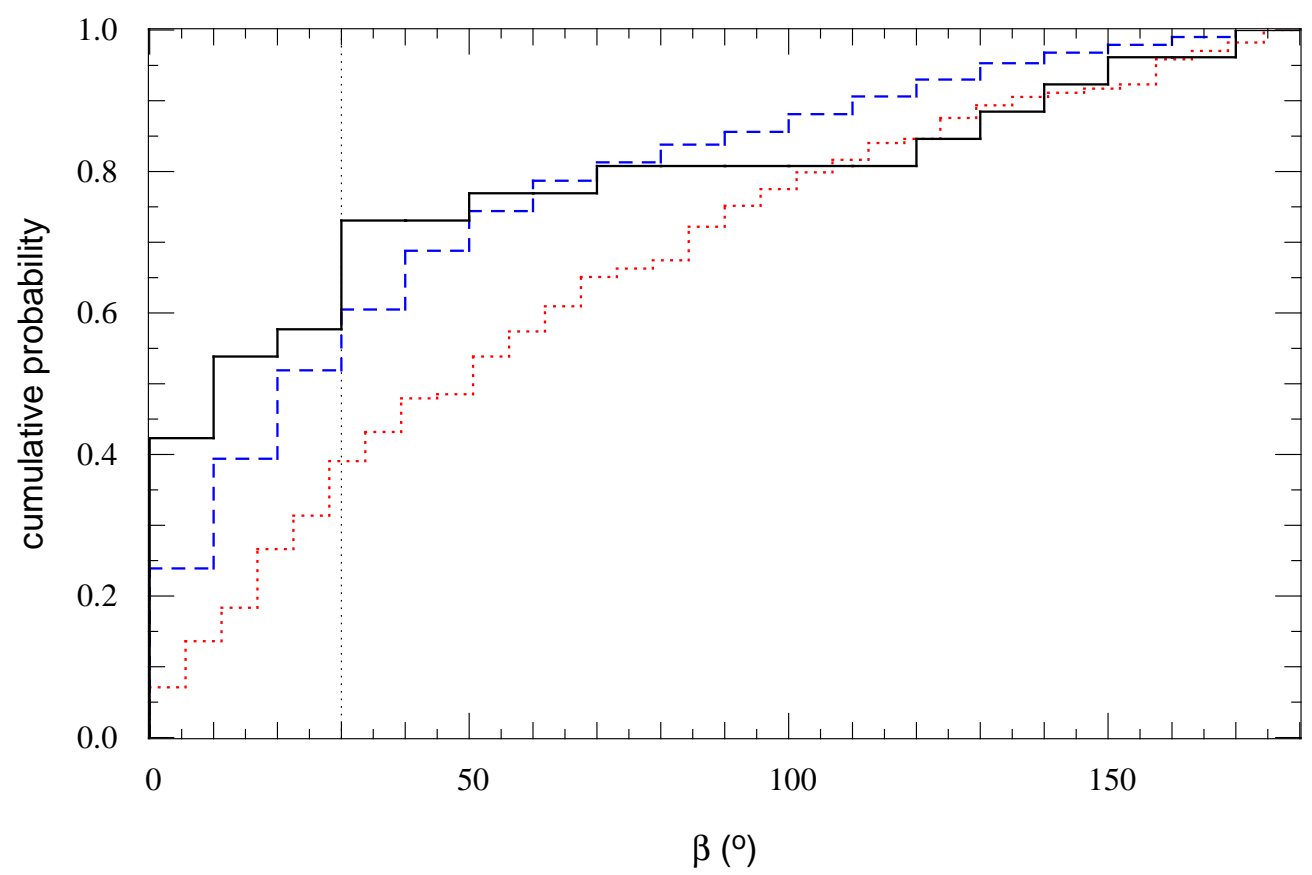

Figure 2: Cumulative distribution of observations (plain black). Theoretical predictions by Fabrycky \& Tremaine (2007) (dashed blue). Theoretical predictions by Nagasawa et al. (2008) (dotted red).

We concluded that standard disc migration, not expected to produce large angles, could not alone produce the observations, that another mechanism ought to be present, the Kozai mechanism fitting best the observations.

These results depended on an assumption: that the probability for a planet to have a certain $\beta$ appeared independent of other parameters for all planets, that we had only one distribution. Winn et al. (2010) showed it was not so by plotting the projected spin-orbit angle in function of the effective temperature of the star (see Josh Winn's contribution).

\section{New results}

Since, we continued observing with HARPS, while other colleagues continued their observations. Combining announcements made during the conference and those that we have in stock we can confirm what Winn et al. (2010) had remarked: planets around stars colder than $6250 \mathrm{~K}$ tend to be more aligned than planets around hotter stars. In fact the combined observations show a large lack of aligned systems for planets around hotter stars compared to those around colder ones (Figure 3). Schlaufman et al. (2010) using another method, independently comes to similar conclusions: planets around more massive stars tend to be more misaligned. 


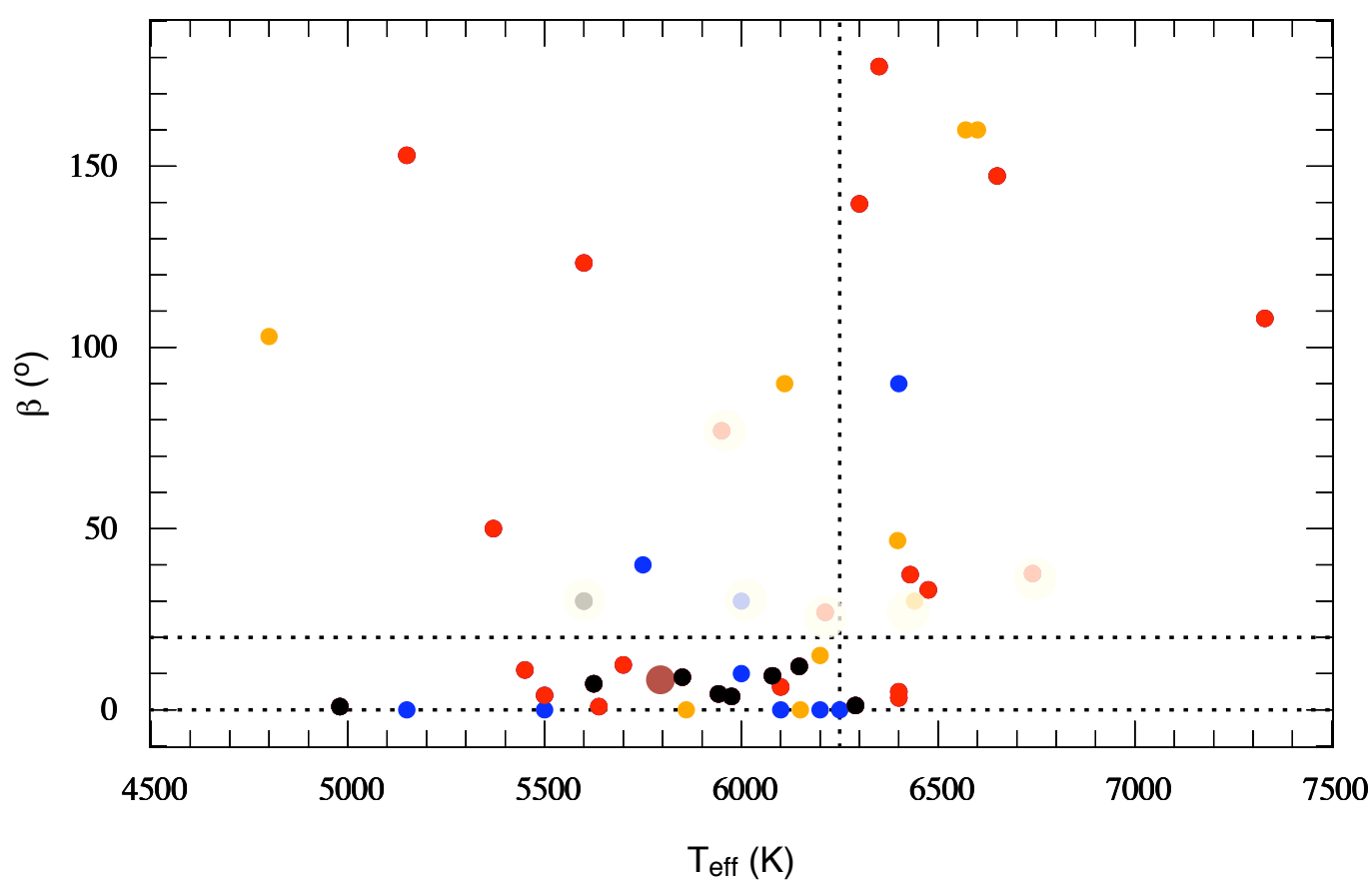

Figure 3: Projected Spin-Orbit angle versus stellar effective temperature. Black $\&$ red symbols: observations prior to the conference. Yellow: observations announced by Faedi, Hébrard, Winn and Narita (see their respective contributions). Blue: HARPS additional observations. Faded symbols: observations which can be doubted. Those are: TReS-1b because of its large error bar, CoRoT-1b, $3 b$ and $11 b$ and Kepler-8b because notably of the bad sampling due to the faintness of those targets. One of our new observations also suffer from some systematic effect. Numbers likely to change.

From explanations given in Winn et al. (2010) $6250 \mathrm{~K}$ is the temperature at which the stellar outer convective envelope disappears. Planets around stars colder than this would realign the convective envelope and therefore would appear aligned; planets around hotter stars retain their original spin-orbit angle. An analysis of the angle of those planets could then be used and compared to theory.

\section{Conclusions}

Although efforts have been made attempting to explain how protoplanetary discs could become misaligned with respect to the central star's spin axis and into which standard migration could happen (Lai, Foucart \& Lin 2010), the lack of aligned systems around hotter stars show that the vast majority of protoplanetary discs ought to be misaligned, something which is not observed (Watson et al. 2010). It leaves multi-body dynamics (placing a planet on an eccentric orbit and tidal friction acting to reduce the semimajor axis) as the only viable solution left. Indeed other indices come to support this view such as how lonely hot Jupiters are. Matsumura, Peale \& Rasio (2010) show that all but five hot Jupiters are located further than 2 Hill radii from the host star, a result 


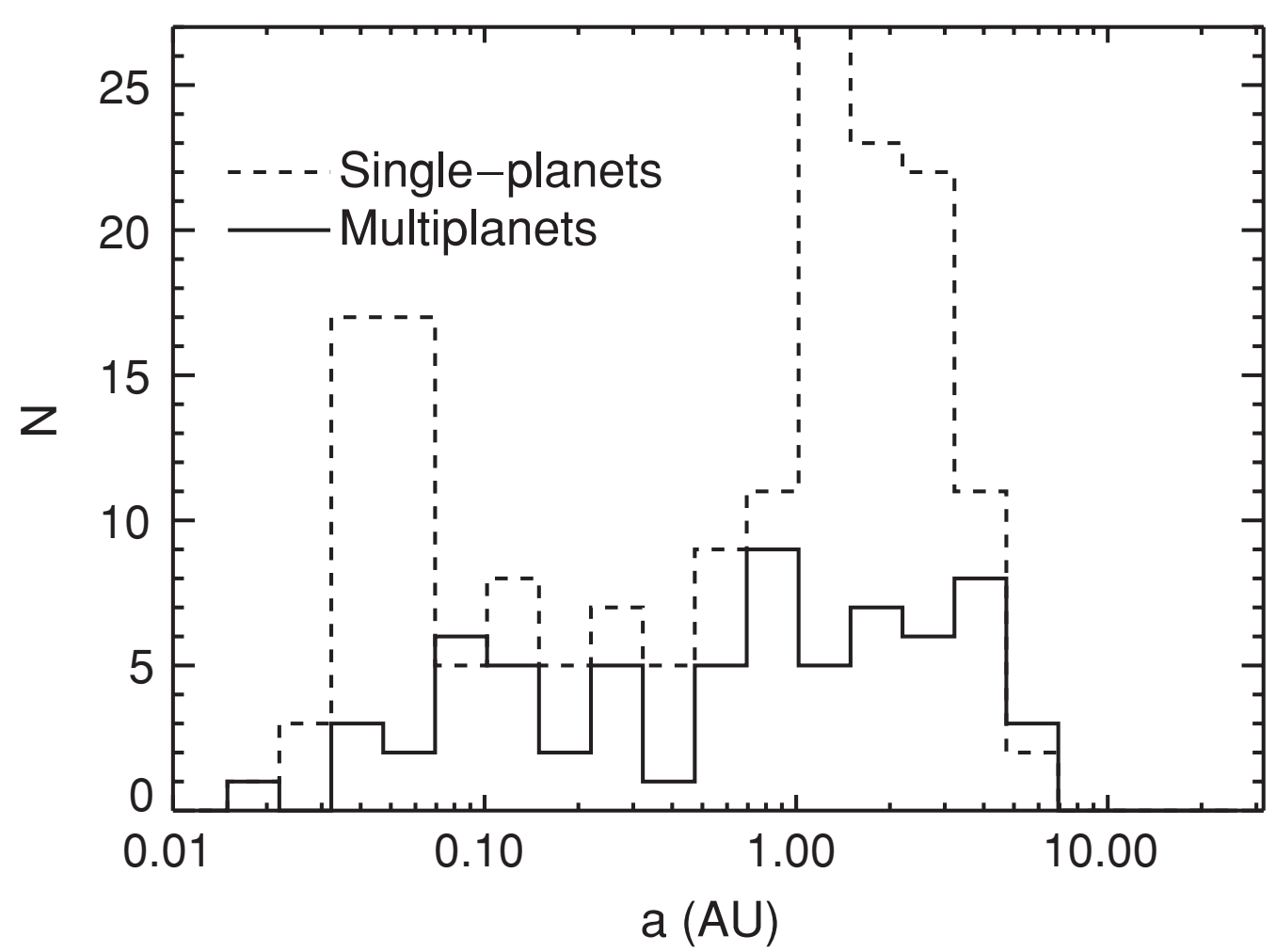

Figure 4: Graph reproduced from Wright et al. (2009).

expected if these had had their orbit circularised from a grazing, highly eccentric orbit. Furthermore, Wright et al. (2009) show that the period distribution for planets is different between single planet systems and multiple systems (Figure 4). One would expect disc migration to deposit planets similarly. This same plot shows a strong rise in the number of planets beyond 1AU. Mordasini et al. (2009), in order to match this $1 \mathrm{AU}$ peak using a population synthesis code, cannot reproduce the hot Jupiter population.

These contributing factors each point towards a dynamical origin for the hot Jupiters, which we remind are only found around about one star in 200. This however should not be interpreted as showing disc migration does not act. There are several indices supporting standard disc migration such as this fore-mentionned rise in planet numbers after 1 AU. Indeed (see Fabrycky's contribution), this is also where the majority of mean motion resonances are found, an expected result of disc migration.

In the scenario we propose disc migration might solely set the initial conditions from which planet-planet scattering or Kozai resonances might act, leading to an orbital evolution ending as hot Jupiter planets. Planets situated between the hot Jupiters and the $1 \mathrm{AU}$ peak might be the result of extreme disc migration, or incomplete dynamics and tidal migration.

We would like to finish by emphasising that these results should also be a call to worry about environmental effects during planet formation and their subsequent 
evolution. It is thought most stars are formed in open clusters. During the time the cluster will live, many stellar encounters can happen and trigger the conditions for Kozai and planet-planet scattering, well after the event occurred (Malmberg et al. $(2007,2010))$.

Acknowledgements. We would like to thank amongst many, D. Fabrycky, D.N.C. Lin, J. Winn, C. Mordasini, S. Matsumura, G. Laughlin, R. Mardling, E. Ford, C. Moutou and G. Hébrard for helpful, interesting and stimulating discussions, notably some taking place at the OHP and all the members of the SOC and of the LOC for making this meeting such a interesting and pleasurable event.

\section{References}

Fabrycky, D., Tremaine, S. 2007, ApJ 669, 1298

Gillon, M., Anderson D.R., Triaud, A.H.M.J. et al. 2009, A\&A 501, 785

Golreich, P., Tremaine, S. 1980, ApJ 241, 425

Holt, J.R. 1893, Astronomy \& Astrophysics XII, 646

Lai, D., Foucart, F., Lin, D.N.C. 2010, submitted

Lin, D.N.C., Bodenheimer, P., Richardon D.C. 1996, Nature 380, 606

Malmberg, D., de Angeli, F., Davies, M.B. et al. 2007, MNRAS 378, 1207

Malmberg, D., Davies, M.B., Heggie, D.C., 2010 submitted

Matsumura, S., Peale, S.J., Rasio, F.A. 2010, submitted

Mayor, M., Queloz, D. 1995, Nature 378, 355

McLaughlin, D.B. 1924, ApJ 60, 22

Mordasini, C., Alibert, Y., Benz, W. et al. 2009, A\&A 501, 1161

Nagasawa, M., Ida, S., Bessho, T. 2008, ApJ 678, 498

Queloz D., Eggenberger, A., Mayor, M. et al. 2000, A\&A 359, L13

Queloz, D., Anderson, D.R., Collier Cameron, A. et al. 2010, A\&A 517, L1

Rasio, F.A., Ford, E. 1996, Science 274, 954

Rossiter, R.A. 1924, ApJ 60, 15

Schlaufman, K. 2010, ApJ 719, 602

Triaud, A.H.M.J., Collier Cameron, A., Queloz, D. et al. 2010, A\&A, in press

Watson, C., Littefair S.P., Diamond, C. et al. 2010, submitted

Winn, J., Fabrycky, D., Albrecht, S. et al. 2010, ApJ 718, L145

Wright, J., Upadhyay, S., Marcy, G.W. et al. 2009, ApJ 693, 1084 